\title{
Logistics, traffic and transportation
}

\author{
Karl F. Doerner • Dennis Huisman • Leena Suhl
}

Published online: 15 July 2014

(C) Springer Science+Business Media New York 2014

Traffic and transportation are of essential importance for the global economy and society. Growing need of transportation and limitation of fossil fuel imply that economical use of transportation resources is critical and will become increasingly important in the future. Simultaneously enterprises are forced to reduce costs and save resources to increase their competitiveness in the global markets. Optimization models and computational solution methods have been developed to manage and control complex systems arising in traffic, transportation and logistics. However, such problems often become very difficult and cannot be solved to optimality with today's technologies. New models and methods are needed to be able to compute better solutions for practical use. The modeling and solution approaches are often based on mathematical programming and complemented with heuristic methods.

The primary objective of this special issue is to examine the methodological and research issues in optimization of logistics, traffic and transportation systems, focusing on the latest developments in modeling and optimization methods. For this special issue six articles have been selected for publication after a thorough peerreview according to the standards of the FSM journal.

\section{K. F. Doerner}

Department of Business Administration, University of Vienna, Oskar-Morgenstern-Platz 1, 1090 Vienna, Austria

e-mail: karl.doerner@univie.ac.at

\section{Huisman}

Econometric Institute, Erasmus University Rotterdam, P.O. Box 1738, 3000 DR Rotterdam, The Netherlands

e-mail: huisman@ese.eur.nl

L. Suhl (西)

DS\&OR Lab, Department of Business Information Systems, University of Paderborn, Warburger Str. 100, 33098 Paderborn, Germany

e-mail: suhl@uni-paderborn.de 


\section{Papers in the special issue}

The first paper by Rune Larsen, Marco Pranzo and Andrea D'Ariano addresses susceptibility of optimal train schedules considering stochastic disturbances and their effects in railway traffic. The research question is to assess the degree of sensitivity of various rescheduling algorithms to variations in process times. Computational results are based on a complex and densely occupied Dutch railway area where train delays are computed based on statistical distributions, and dwell and running times of trains are subject to additional stochastic variations.

Delay management and train scheduling have been studied by Twan Dollevoet, Francesco Corman, Andrea D'Ariano and Dennis Huisman in the second paper as well. Delay management determines which connections should be maintained in case of a delayed feeder train. The authors propose an optimization approach that iteratively solves a macroscopic delay management model on the one hand, and a microscopic train scheduling model on the other hand. The macroscopic model determines which connections to maintain and proposes a disposition timetable. This disposition timetable is then validated microscopically for a bottleneck station of the network, proposing a feasible schedule of railway operations. The iterative optimization framework is evaluated using real-world instances around Utrecht in the Netherlands.

The distribution process of heating oil typically involves solving vehicle routing problems on a daily basis. The paper written by Eric Prescott-Gagnon, Guy Desaulniers and Louis-Martin Rousseau presents heuristic methods for such an oil delivery vehicle routing problem. The authors consider rich vehicle routing problems that may involve heterogeneous vehicle fleet, multiple depots, intra-route replenishments, time windows, driver shifts and optional customers. Three metaheuristics have been developed, namely, a tabu search algorithm, a large neighborhood search (LNS) heuristic based on the tabu search heuristic, and another LNS heuristic based on a column generation heuristic. Computational results obtained on instances derived from a real-world dataset are presented.

The paper by Tiantang Liu, Zhibin Jiang and Na Geng studies the multi-depot heterogeneous fleet capacitated arc routing problem (MDHCARP). This is a problem with rather rare research in the past, but with many applications in real life. The MDHCARP extends the capacitated arc routing problem by considering both the multi-depot case and limited heterogeneous fleet constraints. The authors propose a genetic local search algorithm for the MDHCARP. Computational results are presented that show the superiority of the proposed algorithm compared to existing approaches.

The fifth article is written by Ilse Louwerse, Jos Mijnarends, Ineke Meuffels, Dennis Huisman and Hein Fleuren, and it studies scheduling movements in the network of an express service provider. Transportation of shipments in the network is organized via depots and hubs, where depots are local sorting centers that take care of the collection and delivery of the parcels at the customers, and hubs are used to consolidate the transportation between the depots. The paper addresses the scheduling of all movements in an express network at minimum cost. The problem 
is divided into two subproblems, and a column generation approach and a local search algorithm are used to solve them.

The scheduling of passenger elevators in a building is an online optimization problem, where the data describing the optimization task become available over time. The paper by Benjamin Hiller, Torsten Klug and Andreas Tuchscherer in the special issue considers scheduling of elevator groups such that short waiting and travel times for the passengers are obtained. The authors present an exact reoptimization algorithm that uses column generation techniques and adopts a Branch \& Bound method to solve the column generation problems.

\section{Concluding remarks}

This special issue has greatly benefited from the cooperation among the authors, reviewers, and editors. We would like to express our sincere thanks to the reviewers for their excellent and timely refereeing. Last, but not least, we thank all authors for their contributions which made this special issue possible.

Karl F. Doerner is a professor of production and logistics at the University of Vienna, Austria and head of the Christian Doppler Laboratory for efficient resource management in intermodal transportation. From 2011 to 2014 he has served as the head of the institute for production and logistics management at the Johannes Kepler University Linz, Austria. He received a PhD degree in 2001 and a habilitation degree in 2007 both from the University of Vienna. He was substitute professor at the University of Hamburg and senior researcher at Salzburg Research. Karl F. Doerner has co-authored more than 70 peer-reviewed scientific publications. His research interests include metaheuristic and hybrid search techniques in the applications logistics, transportation and production planning with special focus on collaboration and multiple objectives.

Dennis Huisman is a part-time associate professor of Logistics and Management Science at Erasmus University Rotterdam. In addition, he is part-time expertise manager logistic processes at Netherlands Railways. He combines both positions after he obtained his $\mathrm{PhD}$ from Erasmus University Rotterdam in 2004. His $\mathrm{PhD}$ thesis dealt with integrated and dynamic vehicle and crew scheduling. His current research focuses on Operations Research models and techniques that can support the planning and operations of a railway operator in relation to complex logistical issues. Dennis Huisman is a co-founder of the Erasmus Center for Optimization in Public Transport (ECOPT). Finally, he was a member of Netherlands Railways' team that won the Franz Edelman Award, also known as the Super Bowl of Operations Research, in 2008.

Leena Suhl is a professor of Information Systems and Operations Research at the University of Paderborn, Germany, and director of the Decision Support \& OR Lab. She received a PhD degree at Helsinki University of Technology (now Aalto University) in 1988 and a habilitation degree at Berlin University of Technology in 2003. She was a visiting scientist at IBM Thomas J Watson Research Center and a visiting professor in Universities in Finland, Poland, USA, China and Korea. Leena Suhl is coordinator of numerous research projects with academic and industrial partners and author of more than 100 publications. Her research interests include optimization systems and decision support systems with applications in traffic, transportation, logistics and production systems. 